\title{
Escala de Identidade Moral: Evidências de Validade para o Contexto Brasileiro
}

\author{
Marilia Mesquita Resende - Universidade de Brasilia, Brasilia, Brasil \\ Juliana Barreiros Porto - Universidade de Brasilia, Brasilia, Brasil
}

\begin{abstract}
Resumo
A identidade moral (IM) pode ser definida como o grau em que a moralidade é importante como parte da identidade de uma pessoa. Esse construto tem apresentado fortes relações com intenções e comportamentos morais, mas sua medida ainda não foi adaptada ao Brasil. Assim, o objetivo deste artigo foi encontrar evidências de validade de construto da escala de IM para o contexto brasileiro. Para tanto, foi realizada a tradução da escala de IM e posterior aplicação online junto com escalas de construtos correlatos em uma amostra de 218 pessoas. Os resultados da análise fatorial e de confiabilidade apontaram uma estrutura bifatorial (internalização e simbolização) com índices satisfatórios para a escala de IM. Foram encontradas relações com amparo, desengajamento moral e jeitinho brasileiro, de acordo com as hipóteses previstas. Conclui-se que o instrumento se constitui como uma medida relevante para identificação de características pessoais relacionadas à moralidade e à ética.
\end{abstract}

Palavras-chave: identidade moral, moral, ética, amparo, validade do teste

Moral Identity Scale: Validity Evidences in the Brazilian Context

\begin{abstract}
Moral Identity (MI) can be defined as the degree to which the morality is important as a part of a person's identity. This construct has shown strong relationships with intentions and moral behavior, but has not yet been adapted to Brazil. Thus, the study intended to find evidences of validity of the MI scale to the Brazilian context. Therefore, the MI scale was translated and applied online along with related constructs scales in a sample of 218 people. The results of the factor analysis and reliability showed a two-factor structure (internalization and symbolization) with satisfactory rates for the MI scale. Significant correlations were found with sympathy, moral disengagement and brazilian jeitinho. Therefore, the instrument is constituted as a relevant measure for identifying characteristics related to morality and ethics.

Keywords: moral identity, moral, ethics, sympathy, test validity
\end{abstract}

Escala de Identidad Moral: Evidencias de Validez para el Contexto Brasileño

\section{Resumen}

Identidad moral (IM) puede definirse como el grado de importancia de la moralidad como parte de la identidad de una persona. Este constructo ha presentado fuertes relaciones con intenciones y comportamientos morales, pero la medida todavía no fue adaptada en Brasil. El objetivo de este artículo fue encontrar evidencias de validez del constructo de la escala de identidad moral (IM) para el contexto brasileño. Para eso, se llevó a cabo la traducción de la escala de identidad moral (IM) y posterior aplicación online junto con las escalas de constructos relacionados en una muestra de 218 personas. Los resultados del análisis factorial y la fiabilidad señalaron una estructura bifactorial (internalización y simbolización) con índices satisfactorios para la escala de IM. Fueron encontradas relaciones con apoyo, desvinculación moral y habilidad brasileña (jeitinho brasileño) de acuerdo con las hipótesis previstas. Para concluir, el instrumento se constituye como una medida relevante para la identificación de características personales relacionadas con la ética y la moral.

Palabras-clave: identidad moral, moral, ética, apoyo, validez del test.

O estudo da ética e da moral se inicia nos primórdios da civilização e se destaca como um tema central em diversos meios, permeando a Religião, a Filosofia, a Antropologia, entre outros. A Psicologia Social também tem investigado temas relacionados à moralidade, como agressão, empatia, justiça e normas (Haidt \& Kesebir, 2010). Recentemente o tema tem sido claramente tratado na área e tem sido influenciado pela nova síntese do campo, em que não há preocupação apenas com o aspecto racional e deliberativo do processo de julgamento moral e tomada de decisão, mas também com o papel dos afetos e de processos intuitivos em julgamentos e ações relacionadas à moral.
A Psicologia Social e do desenvolvimento têm investigado o funcionamento moral e como surge o julgamento moral e ético. Um dos pioneiros na área de moralidade foi Piaget, em que ele define moralidade como um conjunto de regras, na qual o sujeito parte de um raciocínio heteronormativo para um pensamento autônomo (Piaget, 1965). Piaget distinguiu os estágios do desenvolvimento moral e analisou o julgamento moral em crianças. Seguindo a abordagem piagetiana, Kohlberg $(1981,1986)$ propõe suas pesquisas sobre desenvolvimento cognitivo moral. Nessa perspectiva, a moralidade é desenvolvida por estágios ao longo da vida em que o homem deixa de responder apenas conforme 
regras até ter uma reflexão crítica sobre o que é certo ou errado. Observam-se algumas diferenças com a teoria proposta por Piaget, tendo em vista que Kohlberg propõe seis estágios organizados em três níveis - pré-convencional, convencional e pós-convencional.

Entretanto, apesar da forte influência da teoria de desenvolvimento moral de Kohlberg e de Piaget, surgiram diversas críticas a esse modelo. Foi apontado que as perspectivas eram muito simplificadas e generalistas para um conceito complexo como julgamento moral (Narvaez, 2005). Ademais, as pesquisas não encontraram fortes relações entre o estágio moral e o comportamento moral, o que sugere a existência de outros fatores além do raciocínio moral (Stets \& Carter, 2011).

Assim, começaram a surgir abordagens que investigam a identidade moral, apontando-a como um mecanismo autorregulatório que motiva o comportamento moral (Blasi, 1983, 1984, 2005). Para Blasi, o que une o julgamento moral com o comportamento moral seria o self moral. $O$ argumento central é de que, apesar das variações dos aspectos morais para cada indivíduo, quando a moralidade é central para o sujeito, ela possibilita a ação por meio da responsabilidade e da autoconsistência (Blasi, 1984). As estruturas morais estão conectadas indiretamente ao comportamento, ou seja, a ação moral é permeada por uma série de aspectos da identidade que implicam na integridade do self (Blasi, 1983).

Como apontado em revisão sobre o construto, a identidade moral pode ser estudada sob duas perspectivas: a de traço e a sociocognitiva (Shao, Aquino, \& Freeman, 2008). O modelo de traços que aborda a identidade como um conceito central para a compreensão do funcionamento dos processos morais deriva das pesquisas de Blasi (1983, 1984, 2005), em que a identidade moral funcionaria como um conjunto de mecanismos autorregulatórios que estabeleceriam parâmetros de comportamentos morais. Blasi (1984) apresenta três componentes centrais do funcionamento moral: o self moral ou identidade moral, o senso de responsabilidade pela ação moral e a autoconsistência. Neste modelo, é fundamental a coerência entre o self que motiva a ação e o comportamento ético.

Entretanto, também foram apontadas críticas ao modelo de centralidade da identidade moral proposto por Blasi (1984), pois se considera que nem sempre a identidade moral leva ao comportamento, podendo haver uma grande influência de aspectos situacionais (Stets \& Carter, 2011). A identidade moral é definida como o grau em que a moralidade é importante como uma parte da identidade de uma pessoa (Hardy \& Carlo, 2011). Para Hardy e Carlo (2011), nem todo comportamento moral é deliberado e racional, dado que, em diversas situações, esse comportamento é automático, ou seja, os julgamentos morais são construídos rapidamente a partir de uma experiência anterior em que há um processamento das informações de forma não consciente (Reynolds, Leavitt, \& DeCelles, 2010).

De modo a rebater as críticas dentro da perspectiva de Blasi, pesquisadores propuseram utilizar a abordagem sociocognitiva, em que a personalidade moral é compreendida em termos de acessibilidade dos esquemas para o processamento da informação (Narvaez \& Lapsley, 2009). Nesse sentido, Aquino e Reed (2002) propuseram uma medida explícita para identidade moral com base na abordagem sociocognitiva. Para os autores, a identidade moral se caracteriza por um autoesquema que a pessoa tem sobre seu caráter moral (Aquino \& Reed, 2002). Apesar dos vários estudos em identidade moral, poucos exploravam o conceito como o de outras identidades sociais que as pessoas assumem, no sentido de que a identidade moral também poderia funcionar como uma base para identificação social e para a construção de definições sobre si, isto é, a identidade moral de um indivíduo pode estar associada a certas atitudes, crenças e comportamentos (Aquino \& Reed, 2002).

Desse modo, Aquino e Reed (2002) propõem que a identidade moral seria essa autoconcepção sobre traços morais, na qual os autoesquemas podem ser ativados conforme a influência de fatores situacionais. Os autores sistematizam e testam empiricamente seu modelo, encontrando dois fatores: internalização (grau com que os traços morais são centrais para o autoconceito do indivíduo) e simbolização (grau com que esses traços morais são refletidos em escolhas e ações). $\mathrm{O}$ instrumento elaborado pelos autores tem apresentado bons índices psicométricos nas pesquisas em que foi utilizado e tem sido o mais utilizado na área, inclusive cerca de $70 \%$ dos artigos empíricos sobre a centralidade moral utilizam esse instrumento (Jennings, Mitchell, \& Hannah, 2015). Apesar de surgirem críticas quanto à escala, principalmente no que se refere ao fator de simbolização por apresentar ações que não são a melhor expressão de engajamento moral, ela ainda é a medida dominante na literatura de self moral (Jennings et al., 2015).

Em revisão recente, os autores destacam que as pesquisas sobre o self moral têm se focado em explicar 
como a moralidade é internalizada no self de um indivíduo e como a moralidade influencia o afeto e a cognição que geram as decisões e o comportamento (Jennings et al., 2015). Verifica-se que o self moral não é uma variável simples, mas sim um construto complexo que envolve diferentes processos e facetas morais.

As pesquisas que utilizam o conceito de identidade moral vêm encontrando relações significativas com intenção e comportamentos éticos, além de verificarem que essa variável tende a interagir com outras, como tipos de julgamentos morais e fatores situacionais (Aquino, Freeman, Reed, Felps, \& Lim, 2009; DeCelles, DeRue, Margolis, \& Ceranic, 2012; McFerran, Aquino, \& Duffy, 2010; Reynolds \& Ceranic, 2007). Entretanto, ainda se sabe pouco sobre diferenças culturais em identidade moral, as bases neurológicas, o grau em que a identidade moral é estável ao longo das situações e os mecanismos que fazem com que a identidade moral leve à ação (Hardy \& Carlo, 2011). Essas lacunas só podem ser supridas a partir do desenvolvimento de medidas para esse construto.

Em uma revisão recente sobre comportamento ético nas organizações (Treviño, den Nieuwenboer e Kish-Gephart, 2014), os autores destacam que o comportamento ético nas organizações pode ser influenciado por diversos fatores, que podem ser contextuais, individuais e da própria questão ética. Entre os individuais, inclui-se a identidade moral, ressaltando o seu papel dentro do contexto organizacional, dado que é uma variável relevante para se compreender a predisposição a violar as regras e as normas sociais. Nesse contexto, Aquino e Freeman (2012) utilizam o modelo sociocognitivo para explicar que a identidade moral pode ser tanto uma diferença individual como um construto mental que pode ser ativado dependendo da situação e das dicas contextuais. Logo, a identidade moral não é estável, ela se altera na medida em que há estímulo ou não para esse tipo de identidade. No estudo de Aquino, Freeman, Reed, Felps e Lim (2009), os autores alteraram justamente a acessibilidade da identidade moral, demonstrando que, a depender do contexto, essa identidade pode estar mais ou menos ativada.

Considerando a importância do construto para os estudos sobre moralidade, a aceitação e os dados favoráveis da escala de Identidade Moral (IM) (Aquino \& Reed, 2002), este estudo optou por traduzir e buscar evidências para essa escala. Para a construção da escala original, primeiramente, os autores conduziram um survey para averiguar os traços de personalidade que são associados à identidade moral pelas pessoas. A análise de conteúdo reduziu a lista para nove traços. Em seguida, eles verificaram se esses traços eram realmente estímulos para a identidade. Após essa fase de definição dos traços, eles elaboraram os itens com base em estudos anteriores sobre identidade. Os resultados das análises fatoriais exploratórias e confirmatórias forneceram boas evidências de validade.

A validade convergente e divergente também foi testada, assim como a comparação da medida explícita de IM com uma medida implícita - Teste de Associação Implícita. Fizeram ainda uma verificação da rede nomológica do construto e a validade preditiva da escala de IM para efeitos psicológicos e comportamentais, como o autoconceito e o voluntariado. Os resultados apontaram uma estrutura bifatorial da escala - Internalização e Simbolização - com bons índices psicométricos que permitem mensurar a identidade moral do indivíduo.

Portanto, o objetivo desta pesquisa foi adaptar a medida de IM de Aquino e Reed (2002) para o contexto brasileiro, investigando suas evidências de validade de construto. Optou-se por utilizar a medida explícita de identidade moral por ela ser o instrumento mais utilizado nas pesquisas da área (Jennings et al., 2015).

Além de análise da estrutura da escala, optou-se por buscar evidências de validade nomológica. Assim, foram considerados os construtos de atitude imoral, amparo, desengajamento moral e jeitinho brasileiro. Neste estudo, optou-se pelo uso de variáveis avaliadas por instrumentos já traduzidos para o português e que apresentaram evidências de validade em amostras brasileiras.

A atitude imoral (Harding \& Phillips, 1986) envolve a avaliação das pessoas frente a questões desonestas e ilegais, logo os indivíduos que valorizam mais a moralidade julgariam ações desonestas como menos justificáveis (H1). A Escala de Atitude Moral apresentou evidências de validade e já foi aplicada no contexto brasileiro em outros estudos (Ferreira, Fischer, Porto, Pilati, \& Milfont, 2012; Miura, 2012).

$\mathrm{O}$ conceito de amparo se refere à característica da moralidade de preocupação com o bem-estar dos outros (Flores-Battistella, Grohmann, \& Iuva-de Mello, 2014). Logo, quanto mais centralizada e simbolizada a identidade moral, mais a pessoa se preocupa com o bem-estar dos demais (H2). Esse construto foi utilizado na avaliação da rede nomológica original da escala de identidade moral (Aquino \& Reed, 2002). A escala que mede esse construto já apresentou evidências de validade para o contexto brasileiro (Flores-Battistella et al., 2014). 
O desengajamento moral se refere à capacidade das pessoas de construírem justificativas por cometerem atos antiéticos ou imorais de forma a manter o seu autoconceito positivo (Bandura, 1999; Detert, Treviño, \& Sweitzer, 2008). No Brasil, foi elaborada uma medida de justificativas de motoristas para o cometimento de infrações de trânsito baseada na teoria do desengajamento moral, tendo apresentado evidências de validade e bons índices psicométricos (Neto, Iglesias, \& Gunther, 2012). Pesquisas anteriores têm apontado para a relação entre desengajamento moral e identidade moral (Detert et al., 2008; Moore, Detert, Treviño, Baker, \& Mayer, 2012), indicando que indivíduos com a identidade moral mais internalizada e simbolizada irão se desengajar menos moralmente $(\mathrm{H} 3)$.

Por fim, o jeitinho brasileiro se refere a um mecanismo social utilizado pelos cidadãos brasileiros para lidar com as situações difíceis que emergem no cotidiano e para resolução de problemas (Duarte, 2006a, 2006b). Por ser uma característica cultural do brasileiro, considera-se importante verificar a sua relação com o conceito de identidade moral (H4), dado que o jeitinho vem sendo mais associado à corrupção e a comportamentos inadequados (Smith, 2008). Ademais, estudos têm utilizado um instrumento com cenários de jeitinho brasileiro e encontrado bons índices psicométricos (Ferreira et al., 2012; Fischer, Ferreira, Milfont, \& Pilati, 2014; Pilati, Milfont, Ferreira, Porto, \& Fischer, 2011).

Portanto, as hipóteses de pesquisa para comparação com os construtos relacionados teoricamente são:

H1. Identidade moral se correlaciona negativamente com atitude imoral.

H2. Identidade moral se correlaciona positivamente com amparo.

H3. Identidade moral se correlaciona negativamente com desengajamento moral.

H4. Identidade moral se correlaciona negativamente com os fatores corrupção e quebra das normas sociais de jeitinho brasileiro.

Cabe destacar ainda um aspecto relevante nas pesquisas de moralidade e comportamento ético que é a desejabilidade social. Esse viés significa que os participantes podem não responder sempre honestamente porque o seu comportamento errado pode ser percebido como inaceitável socialmente. O problema da desejabilidade social é comum nas pesquisas na área de ética e moralidade pela própria natureza da temática (Wouters, Maesschalck, Peeters, \& Roosen, 2014). Nesse sentido, uma das alternativas apontadas pela literatura para lidar com o viés da desejabilidade social é medir esse construto por meio de instrumento próprio, conforme já realizado em contexto brasileiro (Gouveia, Guerra, Sousa, Santos, \& Costa, 2009). Portanto, é de fundamental importância controlar esse viés, dado que sua presença pode mascarar relações entre variáveis ou criar falsas relações entre as variáveis investigadas.

\section{Método}

\section{Participantes}

Participaram do estudo 218 pessoas, sendo 154 mulheres, média de idade $=27,17$ anos $(D P=10,55) \mathrm{e}$ a maioria possui Ensino Superior (52,3\%). Com relação à renda mensal, grande parte não possui renda própria $(22,5 \%)$ ou recebe de cinco a 15 salários mínimos $(22,5 \%)$. A maioria não possui religião (33\%), é católico $(28,4 \%)$ ou evangélico $(18,8 \%)$.

\section{Instrumentos}

Foi utilizada a versão traduzida e retraduzida da Escala de Identidade Moral (Aquino \& Reed, 2002), composta por 10 itens. A instrução inicial do instrumento foi: "A seguir são apresentadas algumas características que podem descrever uma pessoa: Cuidadosa, compassiva, justa, amigável, generosa, prestativa, esforçada, honesta e gentil. Por um momento, visualize o tipo de pessoa que tem essas características. Imagine como essa pessoa iria pensar, sentir e agir. Quando você tiver uma imagem clara de como essa pessoa seria, responda às seguintes questões de acordo com a escala abaixo." Após essa instrução, foi apresentada uma escala tipo Likert de 1 (discordo totalmente) a 5 (concordo totalmente) e os itens.

Para a adaptação da escala de IM ao contexto brasileiro foram seguidas as diretrizes estabelecidas pela Comissão Internacional de Testes para a tradução e adaptação de testes (Muñiz, Elosua, \& Hambleton, 2013). Primeiramente, foi utilizado o método back-translation, ou tradução reversa, da escala original de identidade moral. A partir dessa tradução reversa, foi avaliada a equivalência semântica entre as retraduções e a escala original. Em seguida, foi realizada a avaliação por dois juízes independentes para verificar inconsistências na escala, e depois uma avaliação semântica com dez sujeitos, de modo a atingir o critério de saturação das respostas.

Para testar a relação entre a identidade moral e sua rede nomológica, foram utilizadas quatro escalas: 1) Escala de Atitudes Morais, traduzida e adaptada da Morally Debatable Behaviors Scale - MDBS (Harding \& 
Phillips, 1986) com quatro itens. Verificou-se que os itens dessa escala não variam culturalmente (Vauclair \& Fischer, 2011). Os participantes tinham que avaliar em uma escala de 10 pontos (1 - nunca justificável; 10 - sempre justificáve) o quão justificável era cada comportamento. O alpha de Cronbach da escala neste estudo foi de 0,61 e o lambda 2 de Guttman de 0,64.

2) Subescala de Amparo da Escala de Aceitação de Bem-Estar (Flores-Battistella et al., 2014) composta por três itens. Os participantes responderam em uma escala de 5 pontos (1-discordo totalmente; 5 - concordo totalmente) o quanto eles concordavam com as afirmações. O alfa de Cronbach e o lambda 2 de Guttman da escala aplicada nesse estudo foram ambos de 0,66.

3) Escala de Desengajamento Moral no Trânsito de Neto, Iglesias e Gunther (2012) com 28 itens. Os autores encontraram a seguinte estrutura fatorial da escala composta por três fatores: Reconstrução da Conduta, Minimização da Culpa e Distorção do Agente da Ação. Os participantes tinham que avaliar em uma escala de 10 pontos (1 - discordo totalmente; 4 - concordo totalmente $)$ o quanto eles concordavam com as afirmações. Os fatores nesta amostra apresentaram os seguintes índices de confiabilidade: Fator 1 - Reconstrução da Conduta com 10 itens $\left(\alpha=0,76 ; \lambda_{2}=0,77\right)$; Fator $2-$ Minimização da Culpa com 10 itens $\left(\alpha=0,75 ; \lambda_{2}=0,76\right)$; e Fator 3 - Distorção do Agente da Ação com oito itens ( $\alpha=$ 0,$\left.67 ; \lambda_{2}=0,70\right)$.

4) Escala de Jeitinho Brasileiro (Ferreira et al., 2012) com 19 cenários. Os participantes deveriam ler cenários em que pessoas se comportavam de maneira a caracterizar jeitinho e avaliar a probabilidade de se comportar como a pessoa descrita em uma escala de 11 pontos (0 - muito improvável; 10 - muito provável). Após análise dos componentes principais, foram encontrados três fatores da escala, conforme apontado na literatura (Ferreira et al, 2012). Os fatores encontrados nessa amostra foram: 1) Corrupção - jeitinho usado para resolver problemas por meios ilícitos, com sete itens $(\alpha$ $\left.=0,87 ; \lambda_{2}=0,87\right) ; 2$ ) Criatividade - a pessoa utiliza de meios criativos e inovadores para solucionar problemas, sem quebrar normas, com cinco itens $\left(\alpha=0,62 ; \lambda_{2}=\right.$ $0,63)$; e 3) Quebra das Normas Sociais - uso do jeitinho para contornar alguma norma social e resolver um problema pessoal, com quatro itens $\left(\alpha=0,67 ; \lambda_{2}=0,67\right)$. Os itens que obtiveram carga fatorial inferior a 0,40 foram retirados da análise, portanto, três itens foram excluídos e a escala se configurou com 16 itens ao final.

Também foi aplicada a Escala Reduzida de Desejabilidade Social (Gouveia et al., 2009) com 20 itens, que foi traduzida e adaptada de Crowne e Marlowe (1960), de modo a controlar o viés da desejabilidade social nas respostas. Nessa escala, o participante deve marcar V (verdadeiro) ou $\mathrm{F}$ (falso) em cada item, de acordo com seu comportamento. Quanto maior a pontuação obtida, maior a tendência a desejabilidade social. O alfa de Cronbach da Escala de Desejabilidade Social nessa amostra foi de 0,57 e lambda 2 de Guttman de 0,60. Por fim, foram solicitados dados sociodemográficos aos respondentes.

\section{Procedimentos}

\section{Coleta de Dados}

Após os ajustes finais do instrumento, a escala foi aplicada junto com as demais por meio de formulário eletrônico do Google Docs. A divulgação do questionário foi feita por listas de e-mails e redes sociais por meio da técnica de bola de neve, em que os participantes iniciais de um estudo indicam novos participantes que, por sua vez, indicam novos participantes e assim sucessivamente, até que seja alcançado o objetivo proposto.

\section{Análise de Dados}

Primeiramente, foram realizadas análises fatoriais exploratórias com as quais se buscou evidências de validade de construto por meio de uma solução fatorial convergente com a teoria. Em seguida, foi investigada a confiabilidade dos fatores resultantes, mediante $\mathrm{o}$ cálculo do alfa de Cronbach e do lambda 2 de Guttman. Por fim, foram realizadas análises de correlação da escala de identidade moral com as outras escalas da rede nomológica. Para todas as análises, foi realizado o controle estatístico da variável de desejabilidade social.

\section{Considerações Éticas}

O projeto de pesquisa foi submetido por meio da Plataforma Brasil ao Comitê de Ética em Pesquisa vinculado à temática. $\mathrm{O}$ projeto foi aprovado em 09/10/2014, número do parecer XXX, CAAE XXX.

\section{Resultados}

Primeiramente, foram verificadas as propriedades psicométricas da Escala de Identidade Moral (IM), por meio da inspeção da matriz de correlações, do cálculo do índice de adequação da amostra (KMO) e do teste de esfericidade de Bartlett. Foram encontrados resultados satisfatórios nessa avaliação, dado que foi identificada presença de correlações significativas entre todos os 
itens da escala, o KMO foi de 0,81 e o teste de esfericidade de Bartlett foi significativo $\left(\chi^{2}=654,42 ; p<0,00\right)$.

Em seguida, identificou-se que a matriz de dados permitia extrair até dois fatores pelo critério de Kaiser (K1), que demanda autovalores (eigenvalues) maiores que 1. Esse resultado foi confirmado tanto pelo método de análise paralela (valor do autovalor empírico de 3,8 maior que o randômico de 1,3 para o Fator 1 e valor do autovalor empírico de 1,64 maior que o randômico de 1,24 para o Fator 2), quanto pelo gráfico de scree plot.

Foram encontrados dois fatores: 1) Simbolização $\left(\alpha=0,74 ; \lambda_{2}=0,75\right)$; e 2) Internalização $\left(\alpha=0,73 ; \lambda_{2}=\right.$ $0,75)$. A estrutura encontrada está conforme apontado na literatura da área (Aquino \& Reed, 2002). O Fator 1 extraído condensou $35,86 \%$ da variância do fenômeno, e o Fator 2 explicou 17,89\% da variância. O item "Ser alguém com essas características é uma parte importante de quem eu sou" foi excluído da análise por ter obtido cargas fatoriais semelhantes nos dois fatores. Isso pode ter ocorrido pelo fato de o item estar ambíguo e, apesar de ser originalmente do fator de internalização, possui uma redação que se assemelha aos dos itens do fator simbolização. Sugere-se a reescrita do item de modo a mais bem adaptá-lo ao fator de internalização.

A estrutura final sugerida para a escala ficou, portanto, com nove itens, sendo que as cargas fatoriais variaram de 0,45 a 0,80 e os coeficientes de precisão foram de 0,77 (alfa de Cronbach) e 0,78 (lambda 2 de Guttman). A Tabela 1 resume os resultados obtidos na análise fatorial da Escala de Identidade Moral, na amostra da pesquisa.

Em seguida, foram realizadas algumas comparações entre os grupos constitutivos da amostra, com o objetivo de verificar diferenças intergrupos na internalização e simbolização da identidade moral. Para tanto, procedeu-se à realização de análises de variância, na qual foram conduzidas comparações das médias de sexo, grau de escolaridade, renda mensal e religião. Foram encontradas diferenças significativas de sexo na subescala de internalização $(t=5,5 ; g l=215 ; p<0,05)$, com homens $(M=3,97 ; D P=0,70$; IC- $95 \%=3,79$ $4,15)$ apresentando menores índices de internalização da identidade moral que as mulheres $(M=4,52 ; D P=$ $0,58 ;$ IC- $95 \%=4,41-4,61)$.

Tabela 1

Resultados da Análise Fatorial da Escala de IM

\begin{tabular}{|c|c|c|}
\hline \multirow[t]{2}{*}{ Itens } & $\begin{array}{c}\text { Fator } 1 \text { - } \\
\text { Simbolização }\end{array}$ & $\begin{array}{c}\text { Fator } 2- \\
\text { Internalização }\end{array}$ \\
\hline & Carga Fatorial & Carga Fatorial \\
\hline $\begin{array}{l}\text { 1. Os tipos de coisas que faço no meu tempo livre (por exemplo, hobbies } \\
\text { e atividades) claramente me identificam como uma pessoa que tem essas } \\
\text { características. }\end{array}$ & 0,83 & \\
\hline $\begin{array}{l}\text { 2. Estou ativamente envolvido em atividades que comunicam para os } \\
\text { outros que eu tenho essas características. }\end{array}$ & 0,70 & \\
\hline $\begin{array}{l}\text { 3. Minha participação como membro de certas organizações transmite aos } \\
\text { outros que sou uma pessoa que possui essas características. }\end{array}$ & 0,60 & \\
\hline $\begin{array}{l}\text { 4. Eu normalmente visto roupas que me identificam como alguém que } \\
\text { possui essas características. }\end{array}$ & 0,59 & \\
\hline $\begin{array}{l}\text { 5. Os tipos de livros e revistas que eu leio me identificam como uma } \\
\text { pessoa que possui essas características }\end{array}$ & 0,48 & \\
\hline 6. Eu me sentiria bem em ser uma pessoa que possui essas características. & & 0,80 \\
\hline 7. Eu desejo fortemente ter essas características. & & 0,74 \\
\hline 8. Ter essas características não é tão importante para mim. (I) & & 0,56 \\
\hline 9. Eu teria vergonha de ser uma pessoa que possui essas características. (I) & & 0,53 \\
\hline
\end{tabular}

$\mathrm{I}=$ Item invertido. Alfa de Cronbach $=0,77 /$ Lambda 2 de Guttman $=0,78$. 
No que se refere à renda, foram encontradas diferenças significativas entre o nível de renda e a simbolização da identidade moral $(F=2,61 ; p<0,05)$. O teste post hoc de Bonferroni $(p<0,05)$ apontou que os respondentes que relataram ganhar de cinco a 15 salários mínimos $(M=3,42)$ tiveram uma média significativamente maior no fator de simbolização que aqueles que não possuem renda própria $(M=2,94)$. Não foram encontradas diferenças significativas para religião e grau de escolaridade entre os níveis de internalização e simbolização da IM.

O passo seguinte foi averiguar a validade nomológica, em que o instrumento deve se correlacionar da forma esperada teoricamente com outras medidas. De modo a atingir esse objetivo, foram conduzidas análises de correlação da Escala de IM com a Escala de Atitudes Morais, a subescala Amparo da Escala de Aceitação de Bem-Estar, a Escala de Desengajamento Moral e a Escala de Jeitinho Brasileiro.

Os resultados das correlações são apresentados na Tabela 2. É possível verificar que tanto internalização quanto simbolização da identidade moral se correlacionaram positivamente com amparo. Observa-se ainda que o fator de reconstrução da conduta de desengajamento moral correlacionou-se negativamente com internalização da identidade moral, ou seja, quanto mais internalizada a identidade moral menos a pessoa se desengaja moralmente. Verificou-se ainda uma correlação negativa e significativa do fator de corrupção do jeitinho brasileiro com a internalização da identidade moral. Por fim, o fator de criatividade do jeitinho brasileiro correlacionou-se positivamente com simbolização e com a identidade moral global.

Controlando-se a variável de desejabilidade social, não foram verificadas diferenças nas correlações entre as escalas da rede nomológica e a de identidade moral. Entretanto, ao se avaliar a correlação entre os construtos, nota-se que há uma correlação positiva entre o fator de simbolização e identidade moral global com a desejabilidade social. Esse dado indica que pessoas com maiores níveis de simbolização da identidade moral procuram ser mais agradáveis e mais socialmente aceitas.

\section{Discussão}

A escala de identidade moral apresentou índices psicométricos satisfatórios na amostra em que foi aplicada com uma estrutura bifatorial composta por internalização e simbolização. A configuração dos componentes da escala foi a mesma encontrada no estudo original de criação do instrumento (Aquino \& Reed, 2002). O instrumento se configurou com nove itens que avaliam o autoconceito do indivíduo sobre seus traços morais. Esse resultado evidencia a adequação da medida, como apontado nas pesquisas internacionais que indicam esse instrumento como o mais robusto (Jennings et al., 2015) e que ele vem trazendo resultados positivos em pesquisas que associam identidade e comportamento moral (Shao et al., 2008).

Tabela 2

Correlações entre a Escala de IM e Outros Construtos Teoricamente Relacionados

\begin{tabular}{lcc}
\hline \multirow{2}{*}{ Variáveis } & \multicolumn{2}{c}{ Correlação Observada } \\
\cline { 2 - 3 } & $\mathrm{I}$ & $\mathrm{S}$ \\
\hline Atitude Moral & $-0,11$ & $-0,04$ \\
Amparo & $0,14^{*}$ & $0,36^{* *}$ \\
DM (Reconstrução da Conduta) & $-0,14^{* *}$ & $-0,07$ \\
DM (Minimização da Culpa) & $-0,01$ & 0,11 \\
DM (Distorção do Agente de Ação) & $-0,04$ & 0,02 \\
JB (Corrupção) & $-0,29 * *$ & $-0,03$ \\
JB (Criatividade) & 0,07 & $0,24^{* *}$ \\
JB (Quebra de Normas) & $-0,06$ & $-0,08$ \\
Desejabilidade Social & 0,08 & $0,18^{* *}$ \\
\hline
\end{tabular}

Nota. I = Internalização; $\mathrm{S}=$ Simbolização; DM = Desengajamento Moral; JB = Jeitinho Brasileiro.

${ }^{*} p<0,05 .{ }^{* *} p<0,01$. 
A hipótese "H1. Identidade moral se correlaciona negativamente com atitude imoral" não foi confirmada, pois não foram verificadas correlações significativas entre a identidade moral e a atitude imoral. Isso pode ter ocorrido devido à escala de atitude moral não ter sido a mais adequada para compor a rede nomológica do construto investigado, tendo em vista que pesquisas anteriores não indicaram relações significativas de atitudes morais com características relacionadas ao self como a IM (Ferreira et al., 2012). Sugere-se a utilização de outros instrumentos correlatos apontados no estudo original (Aquino \& Reed, 2002) para verificar a existência de relação com IM.

A hipótese "H2. Identidade moral se correlaciona positivamente com amparo" foi corroborada. A medida tanto em internalização como em simbolização apresentou correlações positivas com amparo, o que revela que as pessoas com maiores níveis de identidade moral se preocupam mais com o bem-estar das demais, conforme apontado na literatura (Aquino \& Reed, 2002).

A hipótese "H3. Identidade moral se correlaciona negativamente com desengajamento moral" foi parcialmente confirmada. Foi verificada a relação negativa entre o fator de reconstrução da conduta de desengajamento moral e a internalização da identidade moral. Esse dado corroborou o que foi encontrado em estudos anteriores (Detert et al., 2008; Moore et al., 2012), indicando que quanto mais internalizada a identidade moral para o sujeito, menos ele irá se desengajar em situações que envolvam decisões éticas. Entretanto, não foi possível encontrar relações significativas entre o componente de simbolização e o desengajamento moral. Esse dado corrobora a dificuldade em encontrar relações significativas entre a faceta de simbolização e outros comportamentos éticos, conforme já relatado por Jennings, Mitchell e Hannah (2015). Isso pode reforçar as críticas a esse fator da escala que apresenta ações que podem não ser bons representantes do engajamento moral (Jennings et al., 2015).

Por fim, a hipótese "H4. Identidade moral se correlaciona negativamente com os fatores corrupção e quebra das normas sociais de jeitinho brasileiro" foi parcialmente corroborada. Observou-se uma relação negativa e significativa entre corrupção da escala de jeitinho brasileiro e a internalização da identidade moral. Nesse sentido, quanto mais internalizada a identidade moral, menos provável de a pessoa se comportar de forma antiética. A relação entre moralidade e jeitinho brasileiro já tem sido investigada em estudos anteriores e tem demonstrado que as características do jeitinho brasileiro tendem a estimular comportamentos antiéticos (Ferreira et al., 2012; Miura, 2012; Pilati et al., 2011).

Houve ainda uma correlação positiva e significativa entre a simbolização da identidade moral e criatividade da escala de jeitinho brasileiro. Apesar de a literatura destacar que pessoas criativas tendem a se comportar de forma mais antiética (Gino \& Ariely, 2012), não há estudos que apontem para a existência de relação positiva ou negativa entre criatividade e identidade moral.

No que se refere aos dados demográficos, foi observada uma leve diferença na internalização da identidade moral entre homens e mulheres, com as mulheres apresentado um nível superior de internalização. Esse dado condiz com as pesquisas na área de moralidade e comportamento ético (Kish-Gephart, Harrison, \& Treviño, 2010) que indicam que as mulheres tendem a relatar mais atitudes éticas e a realizarem julgamentos mais éticos, apesar desses resultados de diferença de sexo serem controversos na literatura.

As evidências de validade de construto e as correlações com construtos relacionados apontam para a adequação da medida para verificar a moralidade para os indivíduos e, desse modo, subsidiar futuras pesquisas na área, reforçando a contribuição teórica do estudo, que já vem encontrando evidências relevantes em contexto internacional (Jennings et al., 2015). Os resultados são promissores para o instrumento de identidade moral, mas alguns resultados esperados não foram encontrados, o que pode ser devido à qualidade dos demais instrumentos utilizados na rede nomológica. Deve-se destacar que quando foi utilizada a mesma escala da rede nomológica do estudo original, a hipótese foi corroborada.

Outras limitações incluem a coleta de dados online, que pode ter limitado aqueles que participavam da pesquisa e por ter se restringido a apenas uma amostra, o que impossibilitou a condução de uma análise fatorial confirmatória da escala. Conforme já observado na literatura, as pesquisas sobre os temas de moral e ética envolvem fortes vieses como a desejabilidade social (Wouters et al., 2014) Além disso, a medida de identidade moral neste estudo foi apenas explícita, por isso seria interessante realizar uma comparação com uma medida implícita como realizado no estudo original (Aquino \& Reed, 2002).

As pesquisas futuras devem examinar a estrutura fatorial encontrada na escala e averiguar se a estrutura bifatorial com os itens apresentados se confirmam, conforme estudo original de Aquino e Reed (2002). Sugere-se que novos estudos testem as relações com 
construtos relacionados teoricamente, utilizando outras medidas e que sejam realizados estudos confirmatórios da escala. $\mathrm{O}$ instrumento que utiliza a abordagem sociocognitiva (Aquino \& Reed, 2002; Shao et al., 2008) para compreender o fenômeno da identidade moral contribui teoricamente trazendo robustez à área. Desse modo, observa-se uma contribuição tanto teórica como metodológica para os estudos de self moral no contexto brasileiro. Apesar das limitações, os resultados são favoráveis à escala e conclui-se que ela pode ser utilizada como uma medida de variável individual sobre moralidade com aplicações para diferentes pesquisas.

\section{Referências}

Aquino, K., Freeman, D., Reed, A., Felps, W., \& Lim, V. K. G. (2009). Testing a social-cognitive model of moral behavior: The interactive influence of situations and moral identity centrality. Journal of Personality and Social Psychology, 97(1), 123-41. doi: 10.1037/a0015406

Aquino, K., \& Reed, A. I. (2002). The selfimportance of moral identity. Journal of Personality and Social Psychology, 83(6), 1423-1440. doi: 10.1037//0022-3514.83.6.1423

Bandura, A. (1999). Moral disengagement in the perpetuation of inhumanities. Personality and Social Psychology Review, 3, 193-209. doi: 10.1207/ s15327957pspr0303_3

Blasi, A. (1983). Moral cognition and moral action: A theoretical perspective. Developmental Review, 3: 178-210.

(1984). Moral Identity: Its Role in Moral Functioning. Em W. M. Kurtines \& J. J. Gewirtz (Eds.), Morality, Moral Behavior and Moral Development (pp. 128-139). New York: John Wiley \& Sons.

(2005). Moral character: A psychological approach. Em D. K. Laspsley \& F. C. Power (Eds.), Character psychology and character education: 67-100. Notre Dame, IN: University of Notre Dame Press.

Crowne, D., \& Marlowe, D. (1960). A new scale of social desirability independent of psychopathology. Journal of Consulting Psychology, 24, 349-354. doi: 10.1037/h0047358

DeCelles, K. a, DeRue, D. S., Margolis, J. D., \& Ceranic, T. L. (2012). Does power corrupt or enable? When and why power facilitates self-interested behavior.
The Journal of Applied Psychology, 97(3), 681-9. doi: $10.1037 / \mathrm{a} 0026811$

Detert, J. R., Treviño, L. K., \& Sweitzer, V. L. (2008). Moral disengagement in ethical decision making: A study of antecedents and outcomes. The Journal of Applied Psychology, 93(2), 374-391. doi: 10.1037/0021-9010.93.2.374

Duarte, F. (2006a). A Double-Edged Sword: The 'Jeitinho" as an ambiguous concept in the brazilian imaginary. International Journal of the Interdisciplinary Social Sciences, 1(1). Recuperado de http://iji.cgpublisher.com/product/pub.88/prod.24

Duarte, F. (2006b). Exploring the interpersonal transaction of the brazilian jeitinho in bureaucratic contexts. Organization, 13(4), 509-527. doi: 10.1177/1350508406065103

Ferreira, M. C., Fischer, R., Porto, J. B., Pilati, R., \& Milfont, T. L. (2012). Unraveling the mystery of Brazilian jeitinho: A cultural exploration of social norms. Personality \& Social Psychology Bulletin, 38(3), 331-44. doi: 10.1177/0146167211427148

Fischer, R., Ferreira, M. C., Milfont, T. L., \& Pilati, R. (2014). Culture of Corruption? The Effects of Priming Corruption Images in a High Corruption Context. Journal of Cross-Cultural Psychology, 45(10), 1594-1605. doi: 10.1177/0022022114548874

Flores-Battistella, L., Grohmann, M., \& Iuvade Mello, C. (2014). Políticas públicas de bem-estar: Modelo de Ahmed e Jackson no contexto brasileiro. Revista Latinoamericana de Ciencias Sociales, Niñez. Y Juventud, 12(2), 659-673. doi: 10.11600/1692715x.12210111213

Gino, F., \& Ariely, D. (2012). The dark side of creativity: Original thinkers can be more dishonest. Journal of Personality and Social Psychology, 102(3), 445-59. doi: 10.1037/a0026406

Gouveia, V. V, Guerra, V. M., Sousa, D. M. F., Santos, W. S., \& Costa, J. de M. (2009). Escala de desejabilidade social de marlowe-crowne: Evidências de sua validade fatorial e consistência interna. Avaliação Psicológica, 8(1), 87-98. Recuperado de http://pepsic.bvsalud.org/scielo.php?pid=S167704712009000100008\&script=sci_arttext

Haidt, J., \& Kesebir, S. (2010). Morality. Em S. Fiske, D. Gilbert \& G. Lindzey (Eds.), Handbook of social psychology (5th ed., pp. 797-832). Hobeken, NJ: Wiley. 
Harding, S., \& Phillips, D. (1986). Contrasting values in Western Europe: Unity, diversity and change. London, UK: Macmillan.

Hardy, S. A., \& Carlo, G. (2011). Moral identity. Em S. J. Schwartz, K. Luyckx \& V. L. Vignoles (Eds.), Handbook of identity theory and research (pp. 495-513). New York, NY: Springer.

Jennings, P. L., Mitchell, M. S., \& Hannah, S. T. (2015). The moral self: A review and integration of the literature. Journal of Organizational Behavior, 36, 104168. doi: $10.1002 /$ job

Kish-Gephart, J. J., Harrison, D. A., \& Treviño, L. K. (2010). Bad apples, bad cases, and bad barrels: Meta-analytic evidence about sources of unethical decisions at work. The Journal of Applied Psychology, 95(1), 1-31. doi: 10.1037/a0017103

Kohlberg, L. (1981). The Philosophy of Moral Development. San Francisco: Harper and Row.

Kohlberg, L. (1986). A current statement on some theoretical issues. Em S. Modgil \& C. Modgil (Eds.), Lawrence Koblberg: Consensus and controversy (pp. 485546). London, Philadelphia: The Falmer Press.

McFerran, B., Aquino, K., \& Duffy, M. (2010). How personality and moral identity relate to individuals' ethical ideology. Business Ethics Quartely, 20(1), 3556. doi: 10.5840/beq20102014

Miura, M. A. (2012). Entre a Simpatia e a corrupção: Análise das dimensões constituintes do Jeitinho Brasileiro (Dissertação de mestrado não publicada). Universidade de Brasilia. Recuperado de http://hdl.handle. net/10482/10431

Moore, C., Detert, J. R., Treviño, L. K., Baker, V. L., \& Mayer, D. M. (2012). Why employees do bad things: Moral disengagement and unethical organizational behavior. Personnel Psychology, 65, 1-48. doi: 10.1111/j.1744-6570.2011.01237.x

Muñiz, J., Elosua, P., \& Hambleton, R. K. (2013). Directrices para la traducción y adaptación de los tests: Segunda edición. Psicothema, 25(2), 151-157. doi: 10.7334/psicothema2013.24

Narvaez, D. (2005). The Neo-Kohlbergian tradition and beyond: Schemas, Expertise, and Character. Em G. Carlo, \& C. Pope-Edwards (Eds.), Nebraska Symposium on Motivation, Vol. 51: Moral motivation through the Lifespan (pp. 119-163). Lincoln, NE: University of Nebraska Press.
Narvaez, D., \& Lapsley, D. K. (2009). Moral identity, moral functioning, and the development of moral character. Em D. M. Bartels, C. W. Bauman, L. J. Skitka \& D. L. Medin (Eds.), The Psychology of Learning and Motivation (50th ed., Vol. 50, pp. 237-274). Elesvier Inc. doi: 10.1016/S0079-7421(08)00408-8

Neto, I. L., Iglesias, F., \& Gunther, H. (2012). Uma medida de justificativas de motoristas para infrações de trânsito. Psico PUCRS, 43(1), 7-13.

Piaget, J. (1965). The moral judgment of the child. (M. Gabain, Trans.). New York: Free Press (Trabalho orginal publicado em 1932).

Pilati, R., Milfont, T. L., Ferreira, M. C., Porto, J. B., \& Fischer, R. (2011). Brazilian jeitinho: Understanding and explaining an indigenous psychological construct. Revista Interamericana de Psicologia, 45(1989), 27-36.

Reynolds, S. J., \& Ceranic, T. L. (2007). The effects of moral judgment and moral identity on moral behavior: An empirical examination of the moral individual. Journal of Applied Psychology, 92(6), 16101624. doi: 10.1037/0021-9010.92.6.1610

Reynolds, S. J., Leavitt, K., \& DeCelles, K. a. (2010). Automatic ethics: The effects of implicit assumptions and contextual cues on moral behavior. Journal of Applied Psychology, 95(4), 752-60. doi: 10.1037/ a0019411

Shao, R. D., Aquino, K., Freeman, D. (2008). Beyond moral reasoning: A review of moral identity research and its implications for business ethics. Business Ethics Quartely, 18, 513-540. doi: 10.5840/ beq200818436

Smith, P. B. (2008). Indigenous aspects of management. Em P. B. Smith, M. F. Peterson, \& D. C. Thomas (Eds.), The handbook of cross-cultural management research (1st ed., pp. 319-332). Thousand Oaks: SAGE.

Stets, J. E., \& Carter, M. J. (2011). The moral self: Applying identity theory. Social Psychology Quarterly, 74(2), 192-215. doi: 10.1177/0190272511407621

Treviño, L. K., den Nieuwenboer, N. A., \& KishGephart, J. J. (2014). (Un)ethical behavior in organizations. Annual Review of Psychology, 65, 63560. doi: 10.1146/annurev-psych-113011-143745

Vauclair, M. C., \& Fischer, R. (2011). Do cultural values predict individuals' moral attitudes? A

Psico-USF, Bragança Paulista, v. 22, n. 1, p. 75-85, jan./abr. 2017 
cross-cultural multi-level approach. European Journal of Social Psychology, 41, 645-657. doi: 10.1002/ ejsp.794

Wouters, K., Maesschalck, J., Peeters, C. F. W., \& Roosen, M. (2014). Methodological issues in the design of online surveys for measuring unethical work behavior: Recommendations on the basis of a split-ballot experiment. Journal of Business Ethics, 120, 275-289. doi: 10.1007/s10551-013-1659-5

Recebido em: 13/07/2015

Reformulação: 25/11/2015

Aceito em: 06/12/2015

Sobre as autoras:

Marília Mesquita Resende é Doutoranda e mestre pelo programa de pós-graduação em Psicologia Social, do Trabalho e das Organizações da Universidade de Brasília. Trabalha na área de psicologia organizacional e gestão de pessoas. Seus interesses de pesquisas enfatizam os seguintes temas: comportamento ético nas organizações, moralidade e cultura organizacional.

E-mail: marilia.mesquitar@gmail.com

Juliana Barreiros Porto é Doutora em Psicologia (UnB) e professora na graduação e no programa de pós-graduação em Psicologia Social, do Trabalho e das Organizações da Universidade de Brasília. Seus interesses de pesquisa e publicações enfatizam os seguintes temas: jeitinho brasileiro, cultura organizacional, valores pessoais e do trabalho, compatibilidade indivíduo-organização e comportamento organizacional positivo.

E-mail: jbporto@unb.br

\section{Contato com as autoras:}

Universidade de Brasília, Campus Darcy Ribeiro, Departamento de Psicologia Social e do Trabalho. Campus Darcy Ribeiro · Instituto Central de Ciências Sul · Instituto de Psicologia · Sala AT- 013.

Brasília-DF, Brasil

CEP: 70910-900 